\title{
Evaluación interdisciplinaria del sistema estomatognático y de la postura en un sujeto diagnosticado con bruxismo del sueño: Reporte de caso
}

\section{Interdisciplinary evaluation of the stomatognathic and posture systems in patients with sleep bruxism: case report}

\author{
Olga-Patricia López-Soto ${ }^{1}$; Julialba Castellanos-Ruíz'; Lina-María López-Soto'; Yéssica-Paola López-Echeverry \\ Yéssica-Tatiana Quintero-Marín ${ }^{1}$; John-William Cardona-Londoño루 Camilo-Andrés Peña-Chantre ${ }^{1}$
}

Forma de citar: López Soto OP, Castellanos Ruíz J, López Soto LM, López Echeverry YP, Quintero Marín YT, Cardona Londoño JW, et al. Evaluación interdisciplinaria del sistema estomatognático y de la postura en un sujeto diagnosticado con bruxismo del sueño: reporte de caso. Salud UIS. 2020; 52(2): 153-159. doi: http://dx.doi.org/10.18273/revsal.v52n2-2020009 (c) (1)

\section{Resumen}

Introducción: Según la literatura científica el bruxismo del sueño (BS) podría producir alteraciones musculares orofaciales, dentales, cefaleas temporales y alteraciones posturales. Objetivo: Evaluar interdisciplinariamente el sistema estomatognático y la postura de un sujeto diagnosticado con bruxismo del sueño. (BS). Materiales y métodos: Estudio descriptivo de reporte de caso de un sujeto de sexo femenino con BS que se diagnosticó mediante polisomnografía. Las relaciones inter-oclusales estáticas y dinámicas se analizaron mediante montaje de modelos en un articulador semi-ajustable. Se realizó evaluación orofacial aplicando mioescanografía, dinamometría y electromiografía y, evaluación de la alineación postural y la estabilidad mediante el índice de Romberg y las presiones plantares con baropodometría. Resultados: En la polisomnografía se registraron 56 eventos de BS/hora, 86 micro-despertares, 11 hipopneas, una tasa cardíaca de 67 1/min y una oximetría de 95\%. En la masticación hubo actividad marcada en maseteros (Der: $330 \mathrm{uV}$ Izq: $452 \mathrm{uV}$ ) y temporal (Der:418 uV, Izq:221uV) y asimetría en temporales de $34 \%$ y en orbiculares de $33 \%$. Hubo disminución de la fuerza contráctil del masetero $(0.3 \mathrm{lb})$ y compresiva del orbicular $(2.0 \mathrm{lb})$. Se registró miositis en los músculos orofaciales y del cuello. Hubo compromiso postural del hemicuerpo izquierdo, estabilidad durante la posición bípeda estática con índice de Romberg de 0.99 y presión plantar mayor en el pie izquierdo de $68,5 \%$ que en el derecho de $31,5 \%$. Conclusiones: En un caso de BS diagnosticado por polisomnografía hubo alteraciones del sistema estomatognático a nivel dental, de ATM y muscular con alteraciones posturales y de la presión plantar.

Palabras Clave: Postura; Sistema estomatognático; Adulto joven; Grupo de salud interdisciplinario.

1. Universidad Autónoma de Manizalez. Caldas, Colombia

Correspondencia: Olga Patricia López Soto. Universida Autónoma de MAnizalez, antigua Estación del Ferrocarril. Teléfono: 8727272 ext 727. Correo electrónico: sonríe@autonoma.edu.co 


\begin{abstract}
Introduction: According to the scientific literature, sleep bruxism (SB) could produce orofacial muscle, dental, and temporary headaches as well as postural alterations. Objective: To evaluate the stomatognathic system and the posture of an individual diagnosed with sleep bruxism (SB) from an interdisciplinary approach. Materials and methods: Descriptive study of a case report of a female individual. The SB was determined by polysomnography. Static and dynamic inter-occlusal relationships were analyzed by assembling models in a semi-adjustable articulator. An orofacial evaluation was performed by applying myoscanoplasty, dynamometry, and electromyography, and an evaluation of postural alignment and stability was performed using the Romberg index and plantar pressures with baropodometry. Results: In the polysomnography, 132 minutes of MOR stage and 265 of NMOR, 56 events of SB / hour, 86 micro-awakenings, 11 hypopneas, a cardiac rate of $671 / \mathrm{min}$, and an oximetry of $95 \%$ were recorded. In the mastication, there was activity marked in masseters (Right: $330 \mathrm{uV}$ Left: $452 \mathrm{uV}$ ) and temporary Right: 418 $\mathrm{uV}$ and asymmetry of temporal (34\%) and orbicular (33\%). There was a decrease in the contractile force of the masseter $(0.3 \mathrm{lb})$ and compressive force of the orbicular $(2.0 \mathrm{lb})$. Myositis was registered in the orofacial and neck muscles. There was postural involvement of left hemibody, stability during static bipedal position with Romberg Index of 0.99, and greater plantar pressure in the left foot of $68.5 \%$ than in the right foot of 31.5.Conclusions: In a case of SB diagnosed by polysomnography, there were alterations of the stomatognathic system at the dental, TMJ, and muscular levels with postural alterations and plantar pressure.
\end{abstract}

Keywords: Posture; Stomatognathic system; Sleep bruxism; Young adult; Patient care team.

\section{Introducción}

El bruxismo del sueño (BS) se define como un movimiento mandibular involuntario, repetitivo $\mathrm{y}$ rítmico durante el sueño que produce un rechinamiento dental generalmente audible ${ }^{1}$. El BS puede tener consecuencias oro-dentales secundarias como fracturas dentales, alteración en la función oclusal y en la articulación temporo-mandibular, dolor o limitación de los movimientos de los músculos orofaciales y cefaleas temporales al fracaso de los tratamientos periodontales, a la aparición de cracks en los dientes posteriores y al fracaso en tratamientos con implantes dentales y sobre- dentaduras ${ }^{2}$. El BS se diagnostica por un examen polisomnográfico que se considera la prueba diagnóstica definitiva ${ }^{3}$.

La biomecánica entre la cabeza, la columna cervical y los órganos dentarios está generando un amplio debate científico por las diferentes interrelaciones que se dan entre ellas, se han observado problemas posturales en más del $55 \%$ de las personas con algún tipo alteración oclusal $^{4}$. Varias publicaciones han descrito la relación entre la postura de la cabeza con los cambios en el sistema estomatognático relacionados con el bruxismo, como el de Motta, et al. en el $2011^{5}$ y el de Velez et al en el $2007^{6}$; sin embargo, son estudios que presentan diagnósticos sin evidencia de interdisciplinariedad entre la odontología y la fisioterapia.

Debido a que los cambios en la articulación cráneocervical podrían alterar directamente la postura de la cabeza, la alteración en la alineación de la articulación occipito-atlanto-axial tiene el potencial de cambiar los contactos oclusales, forzando una adaptación en la función de los músculos de la masticación orofacial ${ }^{7}$. Según esto, es posible que en el tratamiento de sujetos con bruxismo del sueño que tengan manifestaciones de alteraciones dentales, se deba considerar el estado de la articulación cráneo cervical y la articulación temporo-mandibular (ATM). Esto justifica que se realice un diagnóstico interdisciplinario de estos sujetos. Este reporte de caso tuvo como objetivo evaluar interdisciplinariamente la fisiología del sistema estomatognático y postural en un sujeto diagnosticado con bruxismo del sueño.

\section{Materiales y métodos}

Sujeto femenino de 22 años de edad, sin prescripción de medicamentos, no fumadora, con niveles altos de estrés, cefaleas frecuentes al despertar localizadas en la región temporal anterior y consciente de apretamiento dental durante la noche. Para el diagnóstico de bruxismo del sueño (BS), se identificaron criterios diagnósticos bucales como facetas de desgaste dental, hipertrofia del músculo masetero en apretamiento voluntario máximo y dolor en los músculos masticatorios y un examen polisomnográfico. El reporte de este caso fue autorizado por el Comité de bioética de una entidad universitaria (acta de 2018). La participante firmó consentimiento informado. 
La polisomnografía (PSG) registró los parámetros neurofisiológicos, cardio-pulmonares y fisiológicos en el curso de las horas del sueño del sujeto utilizando un análisis automático incorporado en el software del polisomnógrafo Cadwell Easy III. El examen muscular orofacial se realizó mediante Mioescanografía, dinamometría y electromiografía. La mioescanografía se realizó utilizando un equipo Pounds Myoescanner, EilcoTechnologyinc, D-926119T8 Hoxt, 1volt=F.S, pt 2500 y un dinamómetro marca Chatillon, N.Y, U.S.A., model CHA IN-10. Para el registro de la actividad eléctrica de los músculos orofaciales (sEMG) se utilizó un equipo Biometrics DataLog MWX8®. La succión se valoró preguntando al sujeto si tenía hábitos de succión digital, labial o de objetos, la deglución mediante la Técnica de Payne y la respiración por medio de la prueba de Rosenthal.

La evaluación de la alineación postural se realizó en vista posterior, anterior y lateral. La estabilidad se evaluó a través del Índice de Romberg con ojos abiertos y cerrados con estabilometría, se empleó la plataforma de baropodometría, y el programa informático "G-Study", marca TS, modelo P-Walk ${ }^{8}$. Se realizó la prueba de límites de estabilidad para evaluar el control voluntario para desplazar su centro de gravedad hacia sus límites de estabilidad ${ }^{9}$.

\section{Resultados}

Al examen dental clínico se identificó un paladar profundo, facetas de desgaste dental, miositis en los músculos masticadores, hipertrofia de los maseteros y ruidos en la ATM acompañados de dolor en la apertura. En el examen realizado en el articulador semi-ajustable se observó un contacto prematuro con deslizamiento en céntrica, sin interferencias en lateralidad y protrusión, clase Angle I y presencia de guía anterior. Se identificó una succión, deglución, respiración y masticación normales, no se encontraron problemas fono-articulares.

El examen polisomnográfico identificó un tiempo total de sueño de $397.5 \mathrm{~min}$ (6.6 horas). El índice de BS fue de 56 episodios por hora (referencia de normalidad: máximo 25/hora) (Tabla 1 ).

La actividad muscular fue valorada según la cualificación propuesta por Basmajian ${ }^{10}$ (Tabla 2). El índice de asimetría se consideró compatible con una función normal hasta un valor de 18\% según Ferrario, et al. ${ }^{11}$ El sujeto registró asimetría en los músculos temporales y orbiculares durante la masticación y la máxima intercuspidación (Tabla 3).
Tabla 1. Resultados polisomnográficos.

\begin{tabular}{|c|c|}
\hline $\begin{array}{l}\text { Duración de la etapa MOR } \\
\text { (movimiento oculares rápidos) }\end{array}$ & 132 minutos \\
\hline $\begin{array}{l}\text { Duración de la etapa NMOR (fase } \\
\text { de sueño lento) }\end{array}$ & 265.5 minutos \\
\hline Etapa1 (adormecimiento) & 16 minutos \\
\hline $\begin{array}{l}\text { Etapa } 2 \text { (bloqueo de las vías de } \\
\text { información sensorial) }\end{array}$ & 180 minutos \\
\hline Etapa 3 (sueño profundo) & 69.5 minutos \\
\hline Micro-desperatares (arousals) & $\begin{array}{c}86 \text { ( } 52 \text { en MOR y } 34 \\
\text { en NMOR) }\end{array}$ \\
\hline $\begin{array}{l}\text { Hiponeas (disminución del flujo } \\
\text { aéreo de magnitud superior al } 50 \% \\
\text { del flujo basal }\end{array}$ & $\begin{array}{l}5 \text { episodios en } \\
\text { NMOR y } 6 \text { en MOR }\end{array}$ \\
\hline $\begin{array}{l}\text { Promedio de la tasa cardíaca } \\
\text { (normal 60-100 latidos/minuto) }\end{array}$ & 67 \\
\hline $\begin{array}{l}\text { Movimientos periódicos de la } \\
\text { extremidades inferiores }\end{array}$ & 55 \\
\hline Oximetría & $95 \%$ \\
\hline $\begin{array}{l}\text { Número total de eventos de } \\
\text { bruxismo durante el sueño }\end{array}$ & 376 \\
\hline $\begin{array}{l}\text { Ïndice de bruxismo ( número de } \\
\text { eventos/hora): normal hasta } 25 / \text { hora }\end{array}$ & $\begin{array}{c}56.8 \\
(49.7 \text { en NMOR y } \\
70.9 \text { en MOR) }\end{array}$ \\
\hline
\end{tabular}

Tabla 2. Actividad muscular durante la masticación.

\begin{tabular}{lc}
\hline Masetero & $\mathbf{X}$ \\
\hline Derecho & 330,06 (marcada) \\
Izquierdo & 452,37 (marcada) \\
Temporal & \\
Derecho & 418,95 (marcada) \\
Izquierdo & $221,26($ moderada) \\
Actividad orbicular & 481,10 (marcada) \\
Actividad buccinador & \\
Derecho & 223,06 (moderada) \\
Izquierdo & 284,83 (moderada) \\
X: promedio medio en microvoltios (uV)
\end{tabular}

Tabla 3. Asimetría muscular durante la masticación y la máxima intercuspidación.

\begin{tabular}{lcc}
\hline \multicolumn{1}{c}{ Condición } & Músculo & Caso(\%) \\
\hline \multirow{3}{*}{ Masticación } & Maseteros & -15 \\
& Temporales & 34 \\
& Buccinadores & 6 \\
Máxima & Orbiculares & -33 \\
intercuspidación & Total & 1 \\
& Maseteros & 5 \\
& Temporales & 30 \\
& Buccinadores & -9 \\
\hline
\end{tabular}


La fuerza muscular según la mioescanografía registró un imbalance muscular orofacial y disminución en la fuerza contráctil del masetero (Tabla 4).

Tabla 4. Fuerza de los músculos orbicular, maseteros y lingual según mioescanografía

\begin{tabular}{lcc}
\hline \multicolumn{1}{c}{ Músculos } & $\begin{array}{c}\text { Valor de } \\
\text { referencia en } \\
\text { libras }\end{array}$ & $\begin{array}{c}\text { Registro de la } \\
\text { paciente }\end{array}$ \\
\hline $\begin{array}{l}\text { Fuerza compresiva del } \\
\text { músculo orbicular de labios }\end{array}$ & $0.2-0.4$ & 0.4 \\
$\begin{array}{l}\text { Fuerza contráctil del M. } \\
\text { masetero derecho }\end{array}$ & $0.4-0.6$ & 0.4 \\
$\begin{array}{l}\text { Fuerza contráctil del M. } \\
\text { masetero izquierdo }\end{array}$ & $0.4-0.6$ & 0.3 \\
$\begin{array}{l}\text { Fuerza extensora del } \\
\text { músculo lingual }\end{array}$ & $0.6-0.8$ & $0,7$. \\
\hline
\end{tabular}

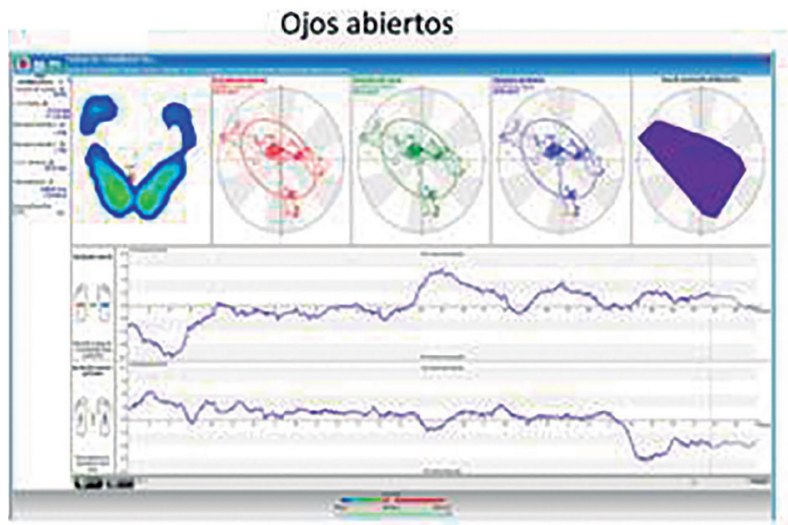

Figura 1. Estabilometría ojos abiertos y ojos cerrados.

Para límites de estabilidad se registró incremento en la amplitud del balanceo con ojos abiertos en sentido anteroposterior y mediolateral. En la frecuencia de
Los rangos de movilidad de la columna cervical alta registraron $25^{\circ}$ de flexión y desde la baja $60^{\circ}$ de flexión. La usuaria manifestó miositis en suboccipital izquierdo, esternocleidomastoideo derecho, suprahioideo izquierdo y bilateralmente en trapecios. En la evaluación postural, en la vista anterior se observó: tendencia a la flexión lateral izquierda de la región cervical, desviación leve de la patela derecha hacia medial, hombro y cresta ilíaca izquierdos descendidos. En la vista posterior: ángulo inferior escapular y espina ilíaca izquierda descendidos. En la vista lateral: cabeza adelantada levemente, protrusión de hombros, pie izquierdo ligeramente plano y pie derecho con arco plantar ligeramente alto.

La estabilometría identificó un índice de Romberg ${ }^{12}$ de 0,99 , indicando una adecuada estabilidad durante la posición bípeda estática (Figura1).

Tabla 5. Límites de estabilidad.

\begin{tabular}{lcccc}
\hline \multicolumn{1}{c}{ Resultados } & \multicolumn{2}{c}{ Ojos abiertos } & \multicolumn{2}{c}{ Ojos cerrados } \\
& Antero-posterior & Medio- lateral & Antero-posterior & Medio-lateral \\
Mínimo & $-56,6$ & $-50,5$ & $-54,8$ & $-36,9$ \\
Máximo & 66,9 & 46,4 & 54,8 & 53,7 \\
Amplitud de balanceo & 40,42 & 38,17 & 28,34 & 32,48 \\
Número de oscilaciones & 23 & 23 & 22 & 15 \\
Tiempo de prueba & 30 & 30 & 30 & 30 \\
Frecuencia de balanceo & 1,30 & 1,30 & 1,36 & 2 \\
\hline
\end{tabular}

La presión media en kilopascales en el pie derecho fue de 25,3 y en el izquierdo de 51,3. El porcentaje de presión fue de 31.5 en el pie derecho y 68.5 en el izquierdo (Figura 2). balanceo se registró un aumento con ojos cerrados. (Tabla 5).

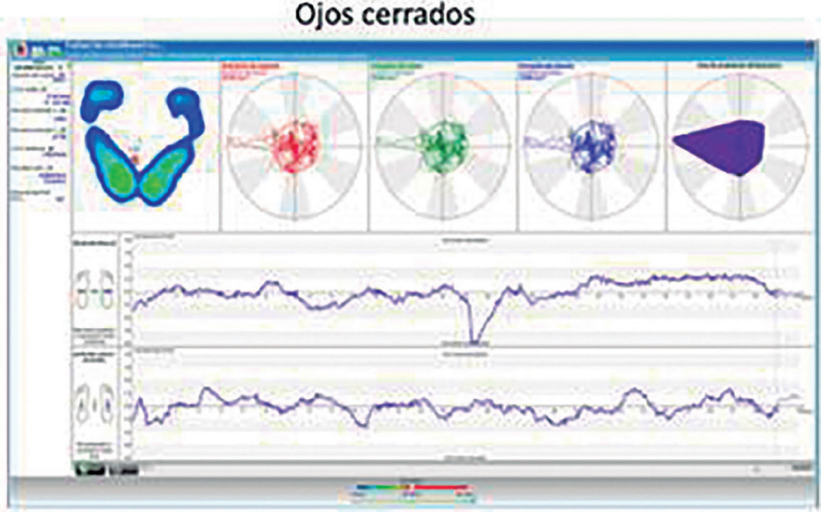

La participante presentó un pie izquierdo ligeramente plano y un pie derecho con un arco ligeramente alto (Figura 3). 


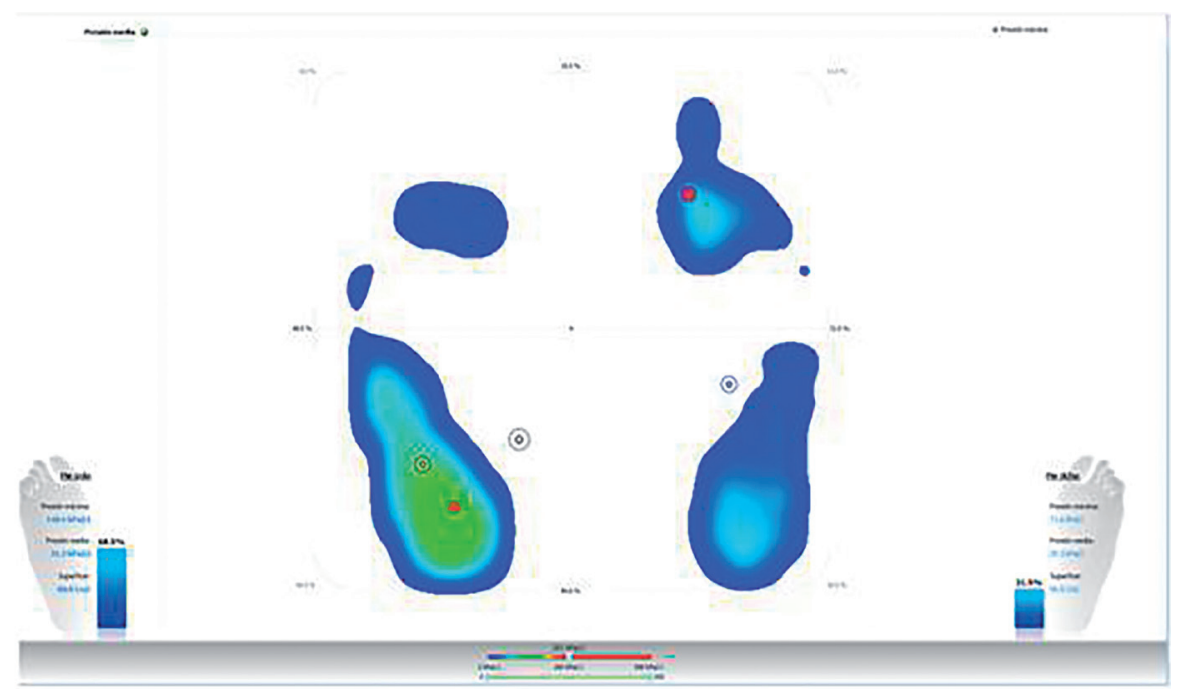

Figura 2. Distribución de la presión plantar.

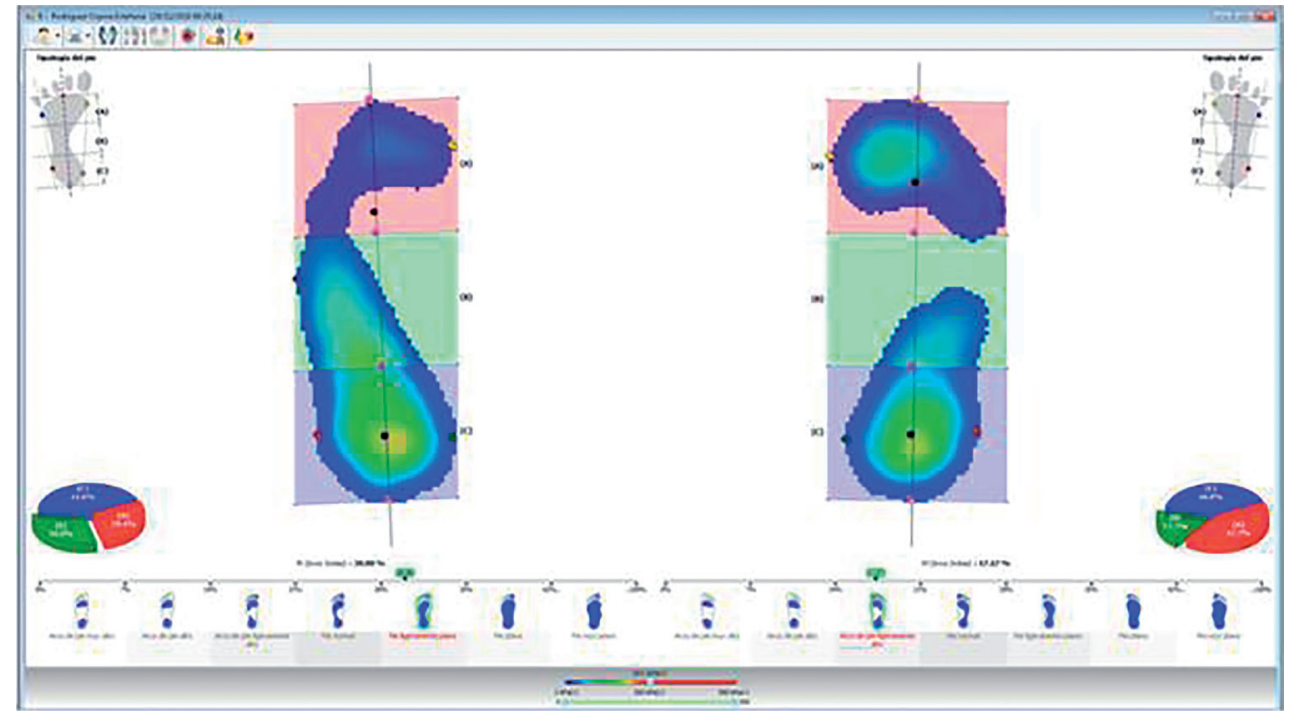

Figura 3. Tipología plantar.

\section{Discusión}

El BS ha sido considerado un factor significativo que aporta sobre-cargas repetitivas sobre el sistema periodontal y la ATM. El bruxismo puede ocasionar una elongación de los ligamentos discales y una incoordinación muscular que produce un movimiento de traslación más amplio de los cóndilos, que explicaría la presencia del ruido $\operatorname{articular}^{13}$. En este reporte de caso hubo actividad "marcada" y asimetría en los músculos masticadores, similar a lo obtenido por Gadott, et al. ${ }^{14}$ quien afirma que la miositis en los músculos masticatorio se relacionaría con las repetidas contracciones musculares a causa del bruxismo de sueño que resultan en fatiga y dolor muscular.
Según Park, et al. ${ }^{15}$ el masetero, bajo contracciones repetidas transmite su tensión al esternocleidomastoideo del mismo lado, la mandíbula se desequilibra, el cráneo toma una posición incorrecta, repercutiendo en la columna cervical por sobrecarga.

La participante registró una alteración en la alineación postural y desplazamiento de centro de gravedad con acortamiento de los músculos extensores de región cervical posterior y del esternocleidomastoideo. Restrepo, et al. ${ }^{16}$ encontraron en un estudio de corte transversal una postura cervical anterior en el grupo con bruxismo. Esta característica según estos autores podría afectar el flujo de aire y volverse parte de la etiología de su para-función. Cuccia, et al. ${ }^{17}$ afirmaron que el 
sistema cráneo-cérvico-mandibular está relacionado con la postura a través de los insumos propioceptivos del cerebelo, núcleos vestibular, oculomotor y colículo superior. Una incorrecta información propioceptiva del sistema estomatognático puede alterar el control de la cabeza y la posición del cuerpo ${ }^{18}$. Una alteración en la posición mandibular puede provocar cambios en las aferencias propioceptivas que pueden diferir la localización del centro de la presión del pie y la estabilidad durante la marcha. En la prueba de baropodometría se presentó mayor presión plantar en el pie izquierdo y mayor compromiso postural en éste hemicuerpo por la presencia de asimetrías. En este caso, el cráneo transfiere su peso a la columna vertebral que puede determinar la distribución de cargas en el antepié y retropié ${ }^{19}$.

En relación con la estabilidad, la usuaria registró un aumento en la frecuencia de balanceos anteroposteriores $\mathrm{y}$ mediolaterales y variaciones con respecto al eje $\mathrm{X}$, Las alteraciones temporo-mandibulares se relacionarían con la distribución de cargas, el centro de gravedad del cuerpo y con la estabilidad ${ }^{19}$.

\section{Conclusiones}

Al realizar la evaluación de sistema estomatognático y de la postura en un sujeto con BS se encontraron alteraciones dentales, en la actividad y simetría muscular orofacial, ruidos en la ATM, miositis en los músculos de cabeza y cuello y alteración postural y de la presión plantar. Esta evaluación demuestra la importancia del trabajo interdisciplinario en los casos de alteraciones en el sistema estomatognático.

\section{Agradecimientos}

Al Laboratorio de Análisis de Movimiento y al Instituto de Idiomas de la Universidad Autónoma de Manizales por el apoyo.

\section{Conflictos de interés}

Los autores declaran no tener conflictos de interés.

\section{Referencias}

1. Klasser G, Rei N, Lavigne G. Sleep bruxism etiology: the evolution of a paradigm. J Can Dent Assoc. 2015; 81: f2.

2. Ekfeldt A, Christiansson U, Eriksson T, Lindén U, Lundqvist S, Rundcrantz T, et al. A retrospective analysis of factors associated with multiple implant failures in maxillae. Clin Oral Implants Res. 2001;12(5): 462-467. doi: 10.1034/j.16000501.2001.120505.x.

3. Carra M, Huynh N, Lavigne G. Sleep bruxism: a comprehensive overview for the dental clinician interested in sleep medicine. Dent Clin North Am. 2012; 56(2): 387-413. doi: 10.1016/j. cden.2012.01.003.

4. Murrieta JF. Maloclusión dental y su relación con la postura corporal: un nuevo reto de investigación en Estomatología. Bol Med Hosp Infant Mex. 2013; 70(5): 341-343.

5. Motta LJ, Martins MD, Fernandes KP, MesquitaFerrari RA, Biasotto-Gonzalez DA, Bussadori SK. Craniocervical posture and bruxism in children. Physiother Res Int. 2011; 16(1): 57-61. doi: 10.1002/pri.478.

6. Vélez AL, Restrepo CC, Peláez-Vargas A, Gallego GJ, Alvarez E, Tamayo V, et al. Head posture and dental wear evaluation of bruxist children with primary teeth. J Oral Rehabil. 2007; 34(9): 663-670. doi: 10.1111/j.1365-2842.2007.01742.x.

7. Westersund CD, Scholten J, Turner RJ. Relationship between craniocervical orientation and center of force of occlusion in adults. Cranio. 2017; 35(5): 283-289. doi: 10.1080/08869634.2016.1235254.

8. Belaguer R. Valoración de un método de posturografía estática con pruebas dinámicas para evaluar funcionalmente pacientes vestibulares en edad laboral y su relación con el índice de discapacidad [Tesis]. Universidad de Valéncia; 2013.

9. De Moya P, Bertomeu B, Broseta V. Evaluación y rehabilitación del equilibrio mediante posturografía. Rehabilitación. 2005; 39(6): 315-323. doi: https:// doi.org/10.1016/S0048-7120(05)74365-6.

10. Basmajian JV. Muscles alive: their functions revealed by electromyography. 1978; 4th edn. Co, Baltimore.

11. Ferrario VF, Sforza C, Miani A Jr, D’Addona A, Barbini E. Electromyographic activity of human masticatory muscles in normal young people. Statistical evaluation of reference values for clinical applications. J Oral Rehabil. 1993; 20(3): 271-280. doi: 10.1111/j.1365-2842.1993.tb01609.x.

12. Ortiz F, Rincón M, Mendoza JC. Texto de medicina física y rehabilitación.1 era Ed. Bogotá, D.C.: Editorial El Manual Moderno Colombia; 2016.

13. Almeida C, Melchior MO, Magri LV, Mestriner W, Oliveira M. Is the Masticatory Function Changed in Patients with Temporomandibular Disorder? Braz Den J 2015; 26(2). doi: https://doi. org/10.1590/0103-6440201300198.

14. Gadotti C, Bérzin F, Gozales D. Preliminary rapport 
on head posture and muscle activity in subjectes with class i and II. J Oral Rehabil. 2005; 32(11): 794-799. doi: 10.1111/j.1365-2842.2005.01508.x.

15. Park B, Tokiwa O, Takezawa Y, Takahashi Y, Sasaguri K, Sato S. Relationship of tooth grinding pattern during sleep bruxism and temporomandibular Joint Status. J Cranio. 2008; 26(1): 8-15. doi: 10.1179/ crn.2008.003.

16. Restrepo CC, Álvarez CP, Jaimes J, Gómez AF. Cervical column posture and airway dimensions in clinical bruxist adults: a preliminary study. J Oral Rehabil. 2013; 40(11): 810-817. doi: 10.1111/ joor.12100.

17. Cuccia A, Caradonna C. The relationship between the stomatognathic system and body posture. Clinics (Sao Paulo). 2009; 64(1): 61-66. doi: 10.1590/ s1807-59322009000100011.

18. Rodríguez C, Torres D, Munuera PV, Pascual ÁO. Influencias de la relación maxilomandibular en el tratamiento postural mediante inhibición muscular. Osteopatía Científica. 2009; 4(3): 115-119. doi: https://doi.org/10.1016/S1886-9297(09)73437-X.

19. Bracco P, Deregibus A, Piscetta R. Effects of different jaw relations on postural stability in human subjects. Neurosci Lett. 2004; 356(3): 228-230. doi: 10.1016/j.neulet.2003.11.055. 